\title{
SUPERVISIÓN DE PH, REDOX Y TURBIDEZ EN UNA PLANTA DE TRATAMIENTO DE AGUA UTILIZANDO WSN (WIRELESS SENSOR NETWORKS) CON TECNOLOGÍA ZIGBEE
}

(Monitoring of PH, redox and turbidity in a water treatment plant using WSN with ZIGBEE technology )

*Gerson Fonseca González, **Eduardo Avendaño, *** Andrés Leonardo Araque

Escuela de Ingeniería Electrónica, UPTC, geriv86@hotmail.com, avendaedu@hotmail.com, andresypm@hotmail.com

(Recibido: el 3 de Febrero de 2014 y aceptado 14 de Mayo de 2014)

\section{Resumen:}

Este artículo muestra el diseño de una WSN utilizando tecnología Zigbee (estándar IEEE 802.15.4). Con ayuda de unos módulos XBee de Maxstream se realiza la comunicación entre una planta de tratamiento de agua de forma remota hacia un nodo coordinador. Los datos adquiridos son ingresados a un microcontrolador, el cual se encarga de procesarlos y formatearlos para poder ser enviados y visualizados bajo una interface gráfica de usuario en Matlab.

Palabras clave: conversión análoga digital, GUI, procesamiento de datos, sensores de $\mathrm{PH}$, redox y turbidez, Zigbee (IEEE 802.15.4).

\section{Abstract:}

This paper presents the design of a WSN using Zigbee technology (standard IEEE 802.15.4). With the help of the XBee of Maxstream modules the communication is carried out between a remote water treatment plant towards a coordinator node. The acquired data are entered to a microcontroller which takes care of the processing to be able to transmit and visualized under a graphical user interface in Matlab.

Keywords: digital analogue conversion, GUI, data processing, PH, Redox and turbidity sensors, ZigBee (IEEE 802.15.4).

\section{INTRODUCCIÓN}

Gracias a los recientes avances tecnológicos, la fabricación de pequeños sensores de bajo costo y consumo de potencia se ha hecho más factible. Las redes de sensores inalámbricos (WSN) están diseñadas para soportar la instrumentación y acondicionamiento de sensores que tomen medidas, por ejemplo, de las condiciones en el ambiente y de acuerdo con sus niveles se informe sobre la dinámica y característica de los mismos.

Las WSN juegan un papel importante en las tecnologías de la información y las comunicaciones (TIC), y están siendo objeto de aplicaciones específicas en todos los campos del conocimiento, debido a su amplio rango de aplicabilidad y su integración con otras tecnologías, entre otras, en la agricultura de precisión, en medicina, en biología, en medicina veterinaria, en control, en domótica, etc.

La supervisión de la calidad del agua es uno de los temas más importantes para la salud de una población. Este documento presenta una forma novedosa de supervisión de algunas variables indispensables para observar el comportamiento de la calidad del agua en plantas de tratamiento, especialmente cuando estas se encuentran a distancias considerables o en lugares en los cuales el acceso es limitado.
De esta manera, las empresas y autoridades pueden de una manera simple tener lectura y tomar acciones preventivas y correctivas sobre los niveles de turbidez, presencia de oxígeno en el agua (óxido-reducción) y PH, y en general si la calidad del agua no es la apropiada, poder estabilizar estos niveles antes de ser distribuida.

\section{MARCO TEÓRICO}

\subsection{Redes inalámbricas de sensores (WSN)}

Las WSN están constituidas por sensores de bajo coste con una interfaz inalámbrica que nos permiten, mediante elementos sensores, recolectar todo tipo de información del mundo físico. Son utilizadas para supervisar de manera cooperativa cualquier fenómeno que pueda ser medido, por ejemplo, condiciones ambientales tales como temperatura, sonido, vibración o, en este caso, agentes contaminantes en las plantas de tratamiento de agua.home automation[ 1 ] [ 3 ] Sin embargo, las redes de sensores inalámbricas son utilizadas en muchas aplicaciones industriales y en zonas civiles, incluidos los procesos industriales de supervisión y control, aplicaciones en salud, automatización del hogar, control de tráfico, del medio ambiente y algunos hábitat y ecosistemas, entre otros.

Las WSN pueden incluir nodos sensores que son, a su vez, actuadores y que permiten controlar diferentes dispositivos utilizando el mismo medio de comunicación inalámbrico.
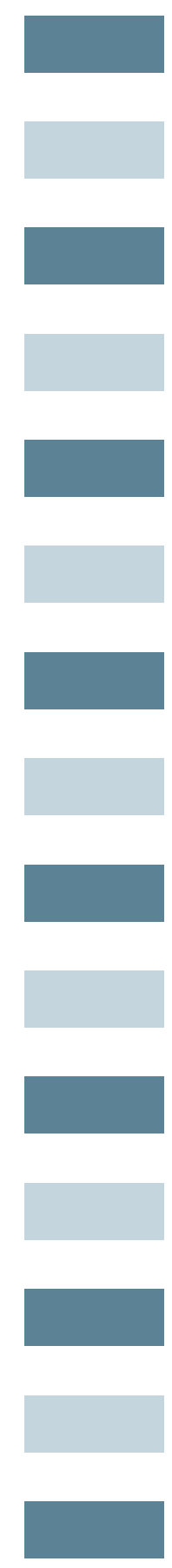
Este último tipo de redes ha acuñado el término redes inalámbricas actuador sensor WSANs. La facilidad y flexibilidad en el despliegue es uno de los principales puntos fuertes que hacen de este tipo de redes un candidato idóneo para un gran número de aplicaciones (ver figura 1). El monitoreo de entornos, control de dispositivos y recolección de datos en bruto son las principales aplicaciones de las WSN y WSAN.

Zigbee es un protocolo de comunicaciones inalámbrico basado en el estándar de comunicaciones para redes inalámbricas IEEE802.15.4, que define el nivel físico y el control de acceso al medio de redes inalámbricas de área personal con tasas de transmisión de datos bajas (low-rate wireless personal area network, LR-WPAN), valores inferiores a $250 \mathrm{Kbps}$.
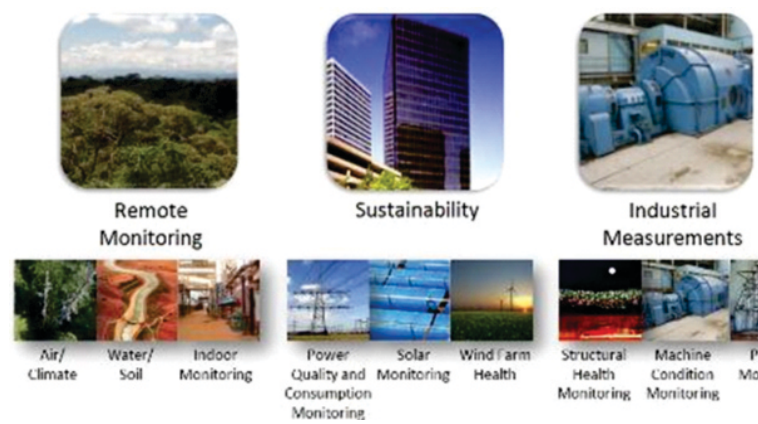
Measurements

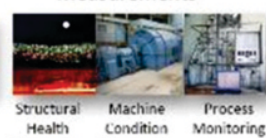

Mealtri Condition Monitoring Monituring Muriturin

Figura 1. Áreas de Aplicación de WSN

\subsection{Norma técnica colombiana para la calidad del agua}

Tiene por objeto establecer los requisitos físicos, químicos y microbiológicos que debe cumplir el agua potable. Esta norma también se aplica al agua potable proveniente de cualquier sistema de abastecimiento (ICONTEC).

\subsection{Definición de parámetros por instrumentar}

A continuación se describen detalladamente las variables por instrumentar para su acondicionamiento de señal.

2.3.1 PH (potencial de hidrógeno). Es un indicador del número de iones de hidrógeno. $\mathrm{El} \mathrm{pH}$ no tiene unidades, se expresa simplemente por un número. Cuando una solución es neutra, el número de protones iguala al número de iones hidroxilo. Cuando el número de iones hidroxilo es mayor, la solución es básica y cuando el número de protones es mayor, la solución es ácida.

El pH del agua puede variar entre 0 y 14 , cuando el $\mathrm{pH}$ de una sustancia es mayor que 7, es una sustancia básica. Ahora, si el pH está por debajo de 7, se denomina sustancia ácida. Cuanto más se aleje el $\mathrm{pH}$ por encima o por debajo de 7, más básica o ácida será la solución (Wikipedia, $\mathrm{pH}$ ).

El sensor de $\mathrm{pH}$ fue implementado teniendo en cuenta su alto costo y la dificultad de comercialización en el país. Por medio de un medidor de $\mathrm{pH}$ industrial, referencia ORION209A+ y su electrodo, se tomaron varias lecturas de pHy el nivel de tensión en el multímetro $(\mathrm{mV})$, los cuales entregaron una diferencia mínima en la lectura, un error del $\pm 2 \%$, que ajustados muestran una linealidad y variación por cada $50 \mathrm{mV}$ por unidad de $\mathrm{pH}$.

2.3.2 Redox. El potencial redox es una medida de la actividad de los electrones. El término redox representa los procesos de reducción y oxidación que ocurren simultáneamente en soluciones acuosas. En un proceso de oxidación, los electrones son transferidos desde la sustancia que va a ser oxidada a la oxidante, es decir, al oxidar la sustancia, el oxidante es reducido. Así mismo, los oxidantes son aceptores de electrones y los agentes reductores son donantes de electrones. Los oxidantes son normalmente cáusticos y los agentes reductores son normalmente acídicos. Este proceso ocupa una posición central en el sistema biológico.

Está relacionado con el $\mathrm{pH}$ y con el contenido de oxígeno. Es análogo al $\mathrm{pH}$, ya que el $\mathrm{pH}$ mide la actividad de protones y el potencial redox mide la de los electrones. El redox se puede calcular de la siguiente manera:

$$
P R=1.234-0.058 p H+0.0145 \log _{10} P_{o}
$$

Siendo $P_{o}$ la presión parcial de oxígeno expresada en atmósferas y su unidad está dada en $\mathrm{mV}$. El potencial es positivo en la zona oxidada y negativo en la zona reducida.

Dado que el redox está relacionado con el pH a través de la fórmula 1, se ingresa el nivel de $\mathrm{pH}$ al microcontrolador $\mathrm{y}$ este se encarga de obtener el valor de oxido reducción.

2.3.3 Turbidez. La turbidez es la expresión de la propiedad óptica de la muestra que causa que los rayos de luz sean dispersados y absorbidos en lugar de ser transmitidos en línea recta a través de la muestra. La turbidez en el agua puede ser causada por la presencia de partículas suspendidas y disueltas de gases, líquidos y sólidos tanto orgánicos como inorgánicos, con un ámbito de tamaños desde el coloidal hasta partículas macroscópicas, dependiendo del grado de turbulencia. En lagos, la turbidez se debe a dispersiones extremadamente finas y coloidales; en los ríos, a dispersiones normales. La eliminación de la turbidez se lleva a cabo mediante procesos de coagulación, asentamiento y filtración (Tripod.com).

La turbiedad merece importante consideración en las aguas para abastecimiento público, por tres razones:

- Estética: cualquier turbidez en el agua para beber, produce en el consumidor un rechazo inmediato y pocos deseos de ingerirla y utilizarla en sus alimentos.

- Filtrabilidad: la filtración del agua se vuelve más difícil y aumenta su costo al aumentar la turbidez.

- Desinfección: un valor alto en la turbidez, es una indicación de la probable presencia de materia orgánica y microorganismos, lo que va a aumentar la cantidad de cloro u ozono que se utilizan para la desinfección de las aguas para abastecimiento de agua potable. 
El límite máximo permisible en el agua potable es de 10 NTU (unidades de turbidez nefelométricas). La unidad de turbiedad fue definida "como la obstrucción óptica de la luz, causada por una parte por millón de sílice en agua destilada", 1 unidad nefelométrica de turbidez (NTU) = $7.5 \mathrm{ppm}$ de $\mathrm{SiO}_{2}$ Actualmente, la unidad utilizada es la NTU, unidad nefelométrica de turbidez y que equivale a 1 unidad nefelométrica de turbidez $(\mathrm{NTU})=1 \mathrm{ppm}$ de formazina estándar.

Este sensor está compuesto por un fototransistor Q1 (OP 802), el cual es enfocado por un laser D1 en su base, y la configuración del fototransistor permite que el voltaje de salida varíe entre 0 y $5 \mathrm{v}$. De esta manera se tienen los voltajes deseados y no es necesario realizar acondicionamiento de señal. Tiene un comportamiento lineal en un rango entre 0 a 100 NTU con variación de voltaje lineal.

\section{DISEÑO DEL PROTOTIPO}

\subsection{Central}

Se construye una estación central o coordinadora compuesta por un computador personal que por medio de una tarjeta con interfaz USB y un módulo con tecnología ZigBee, XBee como se indica en la Figura 2, se encarga de recibir los datos que provienen desde las estaciones remotas, igualmente puede solicitar datos de esta y configurar algunos parámetros sobre la red.
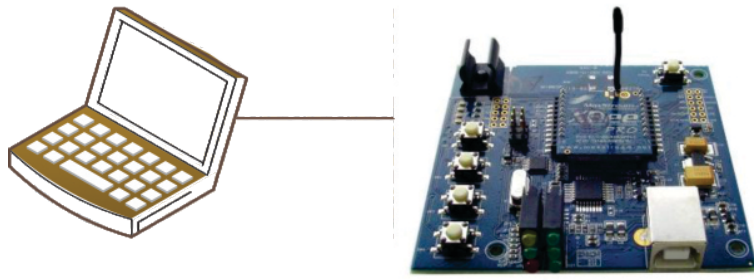

Figura 2. Diagrama estación coordinadora.

\subsection{Estación remota}

En esta se encuentran los sensores, los cuales entran en contacto directamente con las variables por medir. Las señales son adquiridas por medio de un conversor análogo a digital (DAC) en un microcontrolador y procesadas para ser enviadas por medio de la interface aérea del módulo XBee hacia la estación coordinadora.

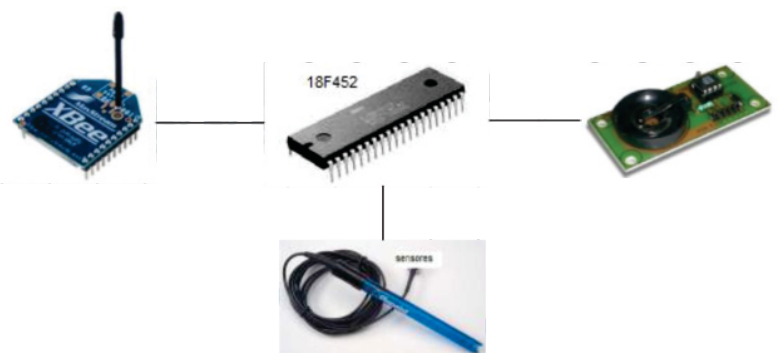

Figura 3. Esquema general estación remota.
La programación del microcontrolador es desarrollada en el compilador PIC C. El diagrama esquemático de la estación remota se muestra en la Figura 4.
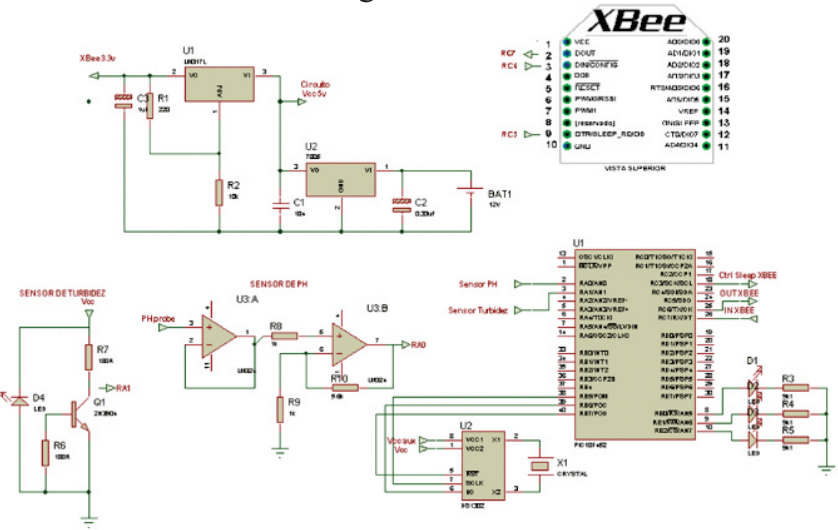

Figura 4. Diagrama esquemático de la estación remota.

\section{ANÁLISIS DE RESULTADOS}

Implementado el prototipo y finalizado el desarrollo de la aplicación, se hicieron algunas pruebas para verificar su funcionamiento.

\subsection{Prueba de funcionamiento}

Para verificar la confiabilidad de la transmisión de los datos obtenidos, el kit de desarrollo incluye un software de programación en comandos AT, denominado X-CTU (software de libre distribución para la configuración de los diferentes módulos XBEE). Para el usuario de la estación central, se diseñó una GUI en Matlab, por medio de la cual se facilita la supervisión del comportamiento de las variables por una representación gráfica (ver Figura 5). Debe tenerse en cuenta que es importante el tiempo de muestreo, dado que los cambios son apreciables en intervalos largos de tiempo. Dentro del menú se puede capturar una medición del estado actual de la variable de interés, la estación coordinadora envía una orden a la estación remota, solicitando los datos actuales de las variables, incluyendo la fecha y la hora en la que se adquirió el dato. Para modificar el periodo de envío de datos de la estación remota, se ingresa el valor deseado en minutos y se selecciona Enviar Tm.

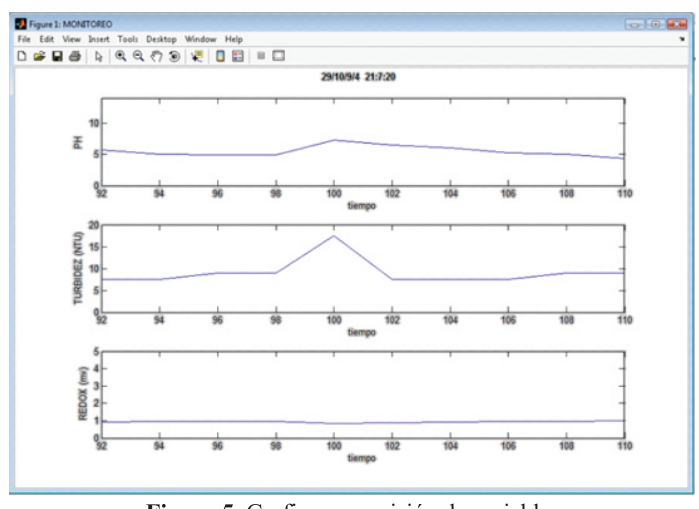

Figura 5. Grafica supervisión de variables

Ahora, a través de un botón denominado Supervisión, se permite realizar una búsqueda de los nodos asociados a la estación central (nodo coordinador).
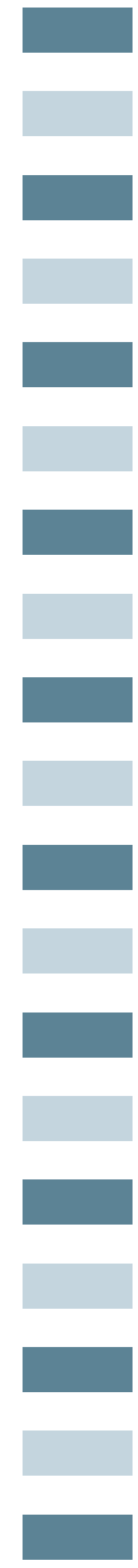
Cuando los datos adquiridos no están en línea, se selecciona el botón Buscar, básicamente la estación central envía una difusión a todos los nodos adicionados a su red, hasta obtener respuesta y estar en línea con la estación remota. En la Figura 7 puede observarse la petición de respuesta emitida por el nodo coordinador, igualmente, si no se encuentran nodos, se despliega un mensaje de tarjeta fuera de rango, indicando que no se logró ninguna comunicación entre las estaciones.
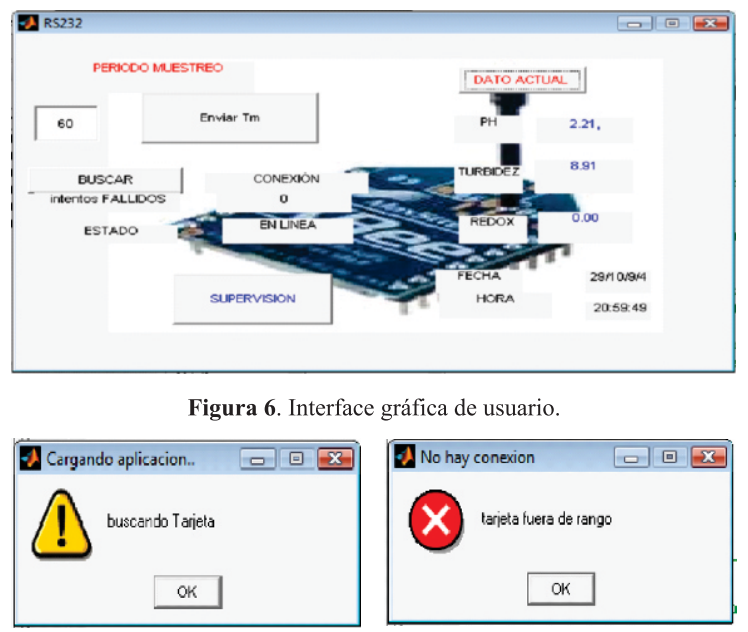

Figura 7. Mensajes de búsqueda y tarjeta fuera de rango.

\subsection{Prueba de calidad del enlace}

Los módulos incluyen parámetros que permiten medir la calidad del enlace. Por medio del software X-CTU se pueden observar dichos parámetros. La calidad del enlace se realizó a campo abierto con visual total entre el transmisor y el receptor. Para evaluar la calidad del enlace se tuvieron en cuenta los siguientes parámetros:

Comando DB (Received Signal Strength): el parámetro de DB se usa para leer la fuerza de la señal recibida (en $\mathrm{dBm}$ ) del último paquete de $\mathrm{RF}$ recibido. Los valores adquiridos se encuentran entre $-40 \mathrm{dBm}$ y la sensibilidad del modulo receptor.

Comando RP(RSSI PWM Timer): este comando es usado para habilitar un PWM (modulado por ancho de pulso) a la salida del módulo. La salida es calibrada para mostrar el nivel de una señal RF recibida y verificar si está sobre el nivel de sensibilidad del módulo. En la Tabla 1 se muestran los resultados obtenidos del enlace en función del rango.

Tabla 1. Distancia, RSSI, potencia
\begin{tabular}{|l|l|}
\hline Distancia (m) & $\begin{array}{l}\text { RSSI } \\
(\mathbf{d B m})\end{array}$ \\
\hline 1 & -42 \\
\hline 10 & -48 \\
\hline 20 & -54 \\
\hline 30 & -56 \\
\hline 40 & -68 \\
\hline 50 & -72 \\
\hline 60 & -80 \\
\hline 70 & -84 \\
\hline 80 & -88 \\
\hline
\end{tabular}

En RSSI, la señal más débil tomada es $-88 \mathrm{dBm}$ y esta ocurre en la distancia máxima.

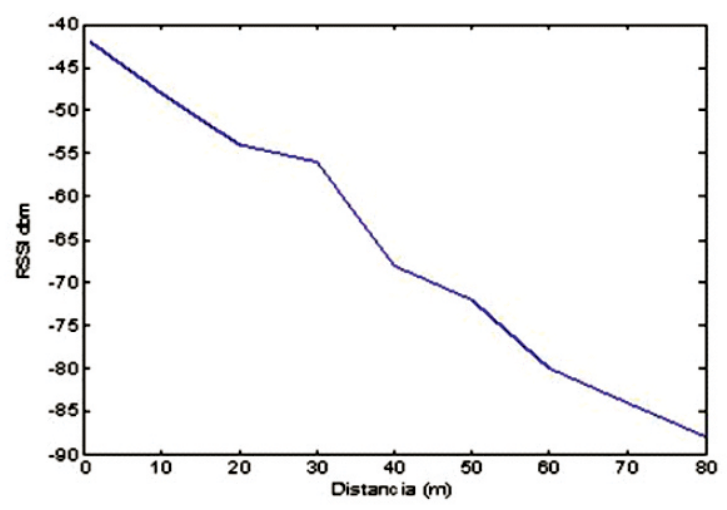

Figura 8. Rango vs. intensidad de la señal recibida (RSSI).

En la Figura 8 se observa que el RSSI disminuye a medida que los dispositivos se están alejando. También se observa que la distancia y el RSSI son directamente proporcionales. Se debe tener en cuenta que los resultados anteriores pueden variar de acuerdo con el entorno en donde se adquieran, dado el ambiente de propagación.

LQI (Link Quality Indication): indica la calidad del enlace, es una medición de la caracterización de la fuerza y/o calidad de un paquete recibido tal como se define en IEEE 802.15.4. El LQI requerido para la norma IEEE 802.15.4 está comprendido entre 40 y $100 \%$, por lo que el sistema de transmisión está cumpliendo con este requisito.

Por medio del analizador de espectro se verificó la señal emitida por la estación coordinadora. Esta se muestra en la Figura 9, en la cual se observa que el lóbulo principal se encuentra en la frecuencia de $2.40907 \mathrm{GHz}$ y con una potencia alrededor de los $-60 \mathrm{dbm}$.

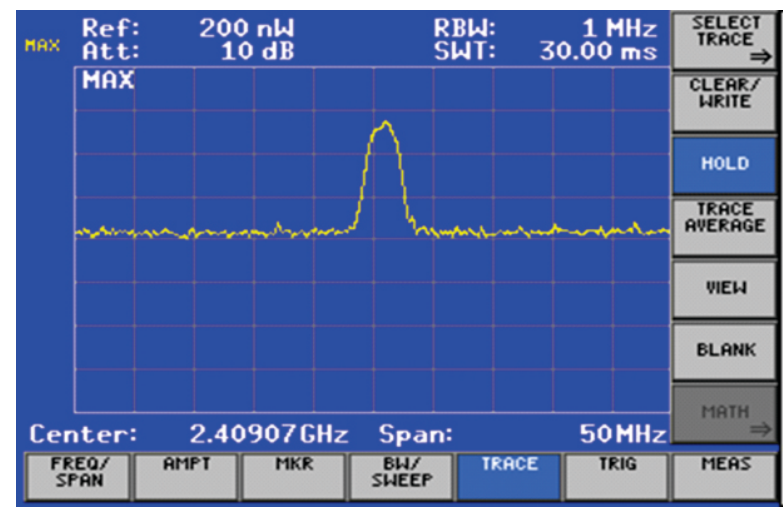

Figura 9. Espectro de la señal transmitida por la estación remota.

\section{CONCLUSIONES}

La supervisión de la calidad del agua es un factor primordial para el cuidado de la salud humana. Con este proyecto se plantea una solución para la supervisión de $\mathrm{pH}$, redox y turbidez, de manera que se puedan conocer sus niveles y, en caso de variaciones significativas, tomar las acciones pertinentes para mantener los niveles óptimos en las plantas de tratamiento. 
La comunicación inalámbrica se realizó satisfactoriamente, usando los módulos XBee. Para obtener mayor alcance de transmisión, se pueden usar los módulos XBee-PRO con antenas externas y mayor potencia de transmisión.

Los sensores implementados en el proyecto ofrecen unos resultados de medición en cuanto a los niveles reales muy cercanos a los resultados arrojados por los sensores usados para calibración. Se recomienda usar sensores reconocidos y especializados para control de calidad del agua.

\section{REFERENCIAS}

Akyildiz, I.F., Weillan, S., Sankarasubramaniam, Y. \& Cayirci, E. (2002, Aug.). A survey on sensor networks. Communication Magazine, IEEE, 40(8).
Chee-Yee, C. \& Kumar, S.P. (2003, Aug.). Proceedings of the IEEE, 91(8).

Ning Xu (2006). A Survey of Sensor Network California: Applications, Computer Science Department, University of Southern California.

Sohraby, K., Minoli, D. \& Znati, T. (2007, March). Wireless sensor networks: technology, protocols, and applications.

Texas Instruments (s.f.). RF/IF and ZigBee ${ }^{\circledR}$ Solutions. $\mathrm{R}$ e $\mathrm{t} \mathrm{r}$ i e $\mathrm{ved}$ f $\mathrm{r}$ o $\mathrm{m}$ http://focus.ti.com/analog/docs/rfifcomponentsho me.tsp?familyId $=367 \&$ content

Wikipedia (s.f.). Sensores. Retrieved from http://www.wikipedia.com/trabajos \Sensores\The Sensor Networks: evolution, opportunities, and challenges. Simplest Possible pH Meter

Zigbee Alliance (s.f.). Retrieved from www.zigbee.org 